\title{
Surgical Techniques for the Management of Enteroatmospheric Fistulae*
}

\author{
Athanasios Marinis, Georgios Gkiokas, Georgios Anastasopoulos, Georgios Fragulidis, \\ Theodosios Theodosopoulos, Thomas Kotsis, Dimitrios Mastorakos, \\ George Polymeneas, and Dionisios Voros
}

\begin{abstract}
Background: An intestinal fistula in the "open abdomen" is called "enteroatmospheric" and is a great challenge for the surgeon because of the high mortality and morbidity rates associated with it. This report is a study of the surgical strategy for treating patients with enteroatmospheric fistulae.

Methods: During a 3-year period (2005-2007), two males and one female patient with a mean age of 63 years were referred to our surgical department for management of enteroatmospheric fistulae that developed after operations carried out for severe peritonitis, which was a consequence of sigmoid diverticulum rupture in two cases and disruption of an entero-enteric Roux-en-Y anastomosis after total gastrectomy for cancer in one.

Results: All patients were appropriately supported in a surgical intensive care unit, with administration of total parenteral nutrition and appropriate antibiotics to eliminate secondary infections. Several re-operations were necessary to treat the enteroatmospheric fistulae. Eventually, all patients were discharged after a lengthy hospital stay (45-145 days).

Conclusions: The essential principles of our operative strategy are: (1) early intervention; (2) a lateral surgical approach via the circumference of the open abdomen to avoid further damage to the exposed viscera; (3) excision of the involved bowel loop with an end-to-end anastomosis; (4) temporary abdominal closure and coverage of the open abdomen with an absorbable mesh, promoting tissue granulation; (5) skin grafting attempts; and (6) selective use of vacuum-assisted closure.
\end{abstract}

D EVELOPMENT OF AN INTESTINAL OPENING from an exposed bowel loop in the middle of the "open abdomen" is called enteroatmospheric fistula and is considered to be one of the most devastating iatrogenic complications of modern surgery. The complexity of managing this problem requires the participation of an experienced surgeon with the advanced support of a surgical intensive care unit (SICU) to treat the ensuing septic complications. In this report, the surgical techniques considered to be critical for the successful outcome of our patients with enteroatmospheric fistulae are presented, and a review of the currently accepted therapeutic strategy is provided.

\section{Case Presentations}

Case 1

A 70-year old man underwent a sigmoidectomy in another hospital for ruptured sigmoid diverticulum. Postoperative peritonitis secondary to rupture of the anastomosis was managed inadequately, resulting in uncontrollable intra-abdominal sepsis and necrotizing infection of the abdominal wall. An open abdomen was created in order to drain pus from the abdominal cavity. After 25 days of supportive care (parenteral nutrition, antibiotics, and care of the open abdomen), exploration revealed erosion of the small bowel, and an enterectomy and end-to-end anastomosis were carried out. However, the hostile abdominal environment led to the development of an enteroatmospheric fistula secondary to rupture of the bowel anastomosis on the eighth postoperative day. Re-operation via a lateral approach was feasible, and resection of the involved small bowel loop and temporary abdominal closure with an absorbable mesh (Vicryl ${ }^{\circledR}$ ) was carried out. Two weeks later, a new enteroatmospheric fistula ensued, and supportive care was instituted for 40 days. Because of the risk of short bowel syndrome, several efforts at local excision of the fistula tract were attempted. The open

*Presented at the Twenty-First Annual Congress of the Surgical Infection Society-Europe, Antalya, Turkey, May 1-3, 2008. 
abdomen was covered once more by an absorbable mesh and finally by an autologous split-thickness skin graft. The patient was discharged after 145 days.

\section{Case 2}

A 39-year old man was admitted urgently to another medical center with neglected fecal peritonitis secondary to ruptured sigmoid diverticulum. A sigmoidectomy and a Hartmann procedure were carried out. Overwhelming sepsis and subsequent multiple organ dysfunction syndrome ensued, with the patient experiencing a lengthy ICU stay. In order to manage ongoing intra-abdominal sepsis, the patient underwent several exploratory laparotomies through an open abdomen. An enteroatmospheric fistula developed, triggering uncontrolled sepsis. Five months after initial admission, the patient was stabilized clinically and was referred to our Surgical Department. Through a lateral surgical approach via the circumference of the open abdomen (Fig. 1A), the involved small bowel loop was readily approached (Fig. 1B), and an enterectomy with an end-to-end anastomosis, cholecystectomy, drainage of a pelvic abscess, and abdominal wound closure were carried out. Unfortunately, three weeks later, wound dehiscence with the development of a new enteroatmospheric fistula occurred. A second operation again using the lateral approach to the open
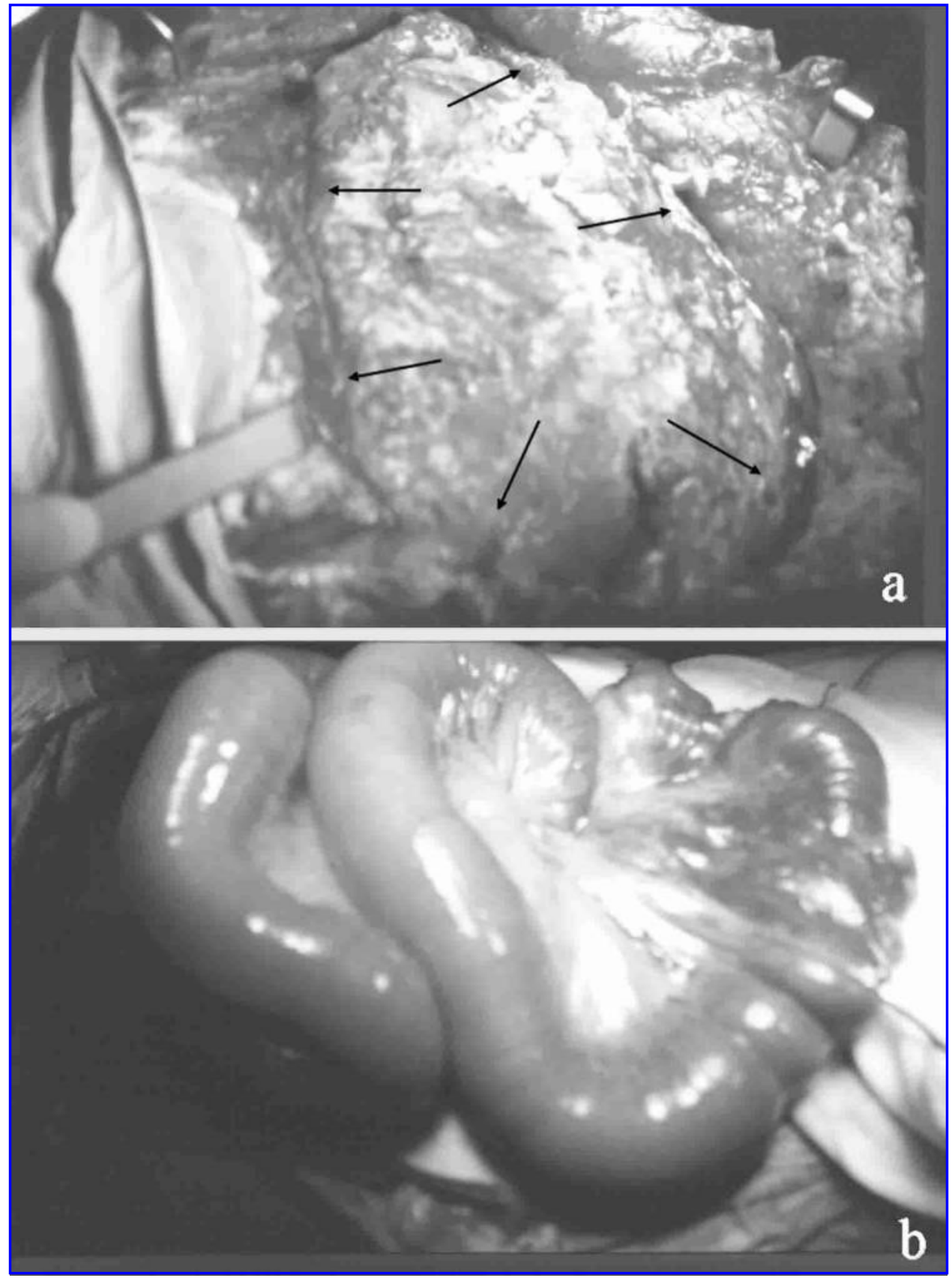

FIG. 1. Surgical technique in case 2. (a) Lateral surgical approach (arrows) leading to safe entrance into abdomen. (b) Involved bowel loop is approached. 
abdomen entailed excision of the involved intestinal loop and end-to-end anastomosis, and temporary abdominal closure was attempted by suturing an absorbable mesh (Vicryl ${ }^{\circledR}$ Ethicon, Inc., Somerville, NJ) to the fascial edges of the abdominal defect. Granulation tissue covered the mesh during the following 3-4 weeks (Fig. 2A). An autologous split-thickness skin graft from the patient's left thigh was placed over the wound (Fig. 2B). Unfortunately, several days after skin grafting a new intestinal fistula occurred. Hopeful efforts to drain intestinal effluents and to promote wound healing using a vacuum-assisted closure (VAC) device were made. Several weeks later a very low output $(<20 \mathrm{ml})$ enterocutaneous fistula was covered by a new split thickness skin graft harvested from the contralateral thigh. Eventually, the fistula was sealed, and the patient gradually re-established a normal diet. The patient was discharged after 133 days and referred to another medical center for orthopedic management of locked joints, kinetic physiotherapy, and physical rehabilitation.

\section{Case 3}

A 78-year old woman with gastric adenocarcinoma underwent a total gastrectomy and Billroth II esophago-jejunostomy. Because of severe postoperative bile reflux, conversion of the primary anastomosis to a Roux-en-Y esophago-jejunostomy was necessary. However, disruption of the jejunojejunal anastomosis resulted in peritonitis and wound dehiscence, which was managed by leaving the abdomen open.

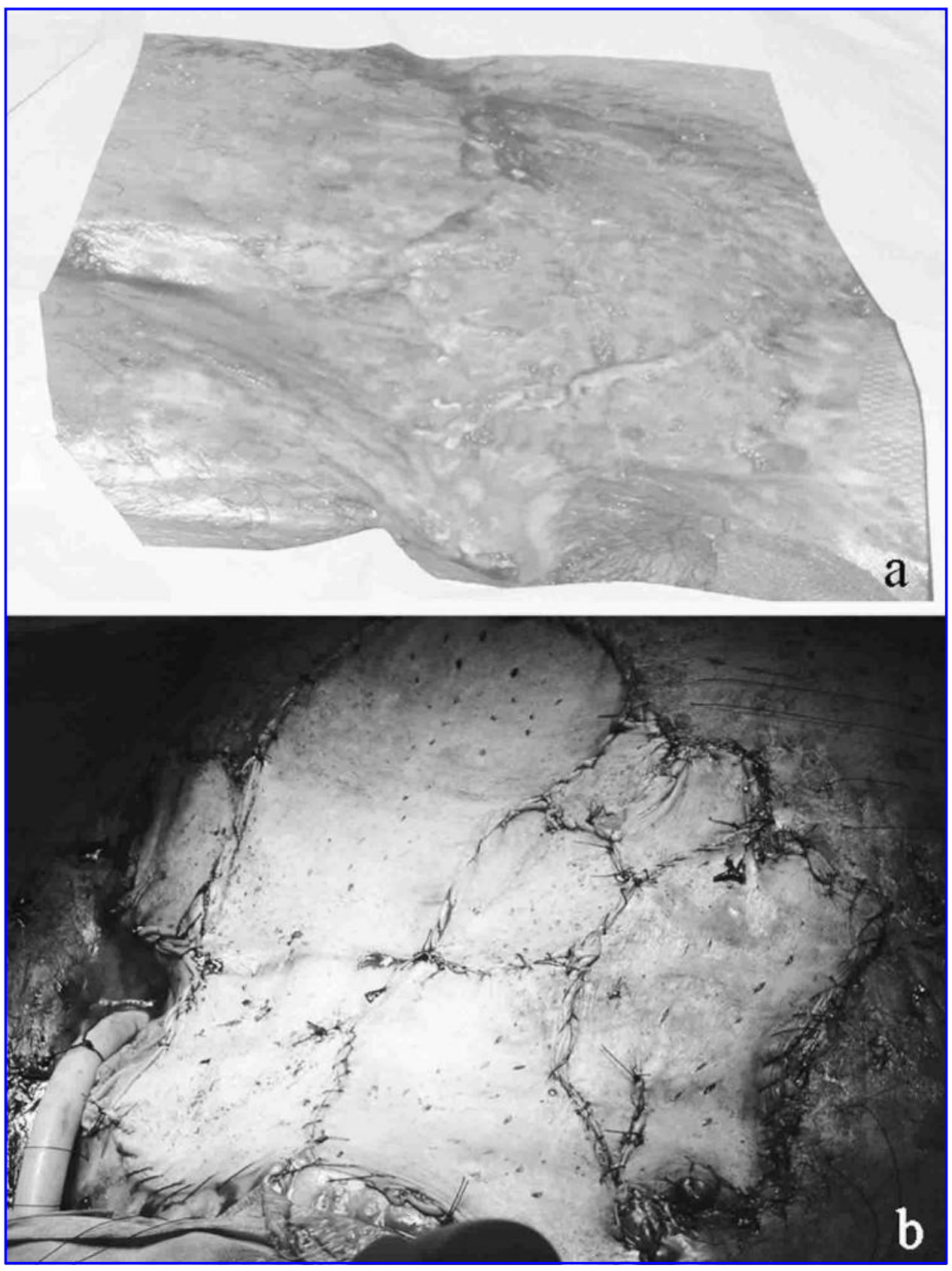

FIG. 2. Surgical technique in case 2 (continued). (a) Temporary abdominal closure with absorbable mesh in development of granulation tissue and (b) application of autologous split-thickness skin grafts. 
Because the fistula tract of this enteroatmospheric fistula was indirect (Fig. 3A), successive applications of a VAC device were made for 12 days (Fig. 3B), which led to the sealing of the fistula and formation of granulation tissue on the abdominal wound. Finally, a skin-only closure was adequate for the coverage of the abdomen. The patient was discharged after 45 days.

\section{Discussion}

"Open abdomen" is a widely accepted therapeutic strategy in circumstances requiring complex surgical intervention, such as severe intra-abdominal infections, acute mesen- teric ischemia, necrotizing infections of the abdominal wall, damage-control surgery for trauma, and abdominal compartment syndrome [1]. Intestinal fistulae in this hostile environment, occurring in approximately $25 \%$ of patients, are a challenging situation for surgeons, being associated with a mortality rate as high as $40 \%$ [2]. Sepsis secondary to peritonitis from spillage of intestinal contents is the major complicating factor. The therapeutic strategy is based on specific principles to overcome the septic complications related to the enteroatmospheric communication and to promote healing and fistula closure [1].

A SICU environment and a multidisciplinary team (senior surgeon, surgeon intensivist, plastic surgeon, physiothera-

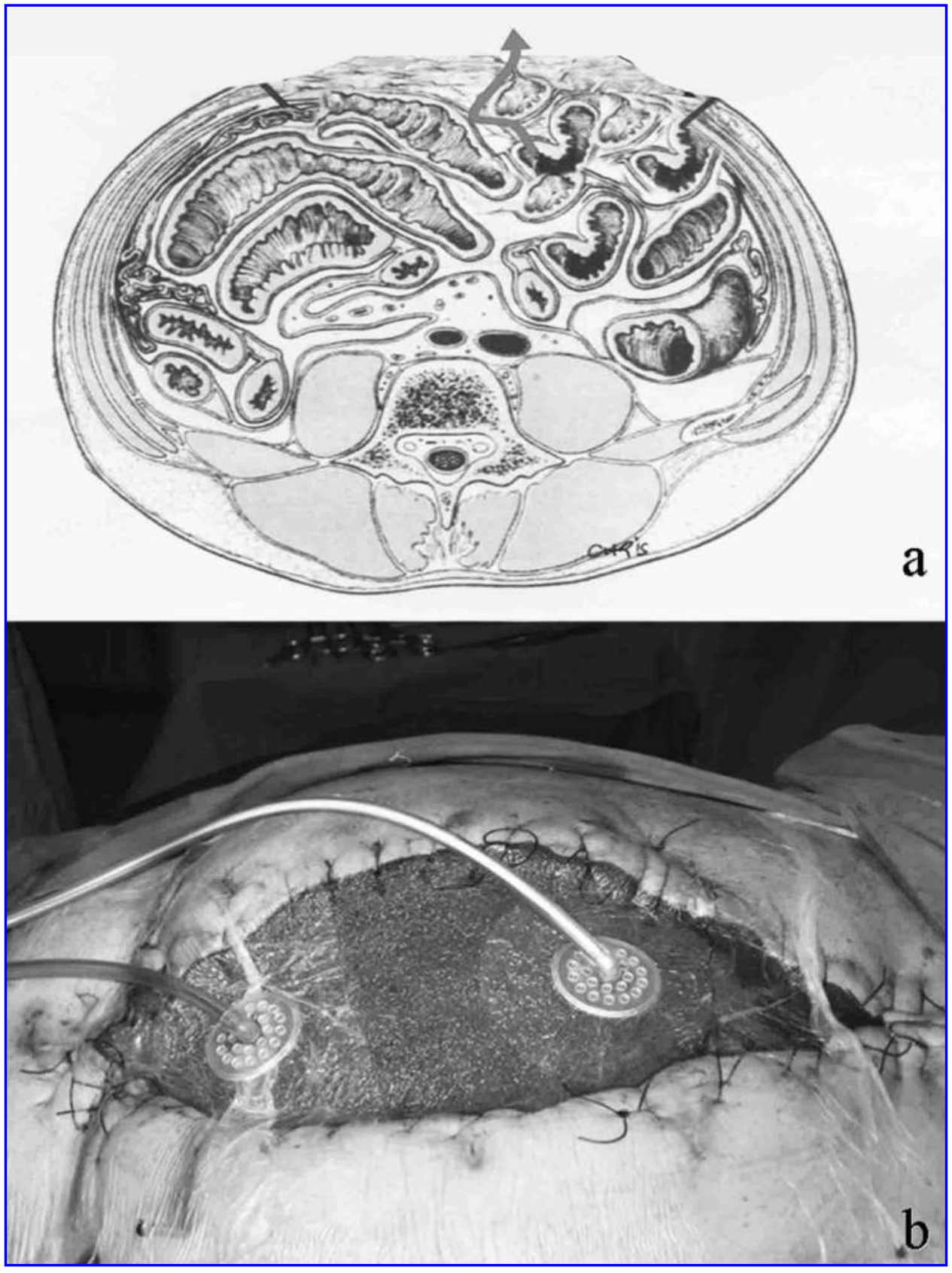

FIG. 3. Surgical technique in case 3. (a) Schematic depiction of indirect course of fistula tract (curved arrow). (b) Use of VAC device. 
$\mathbf{a}$

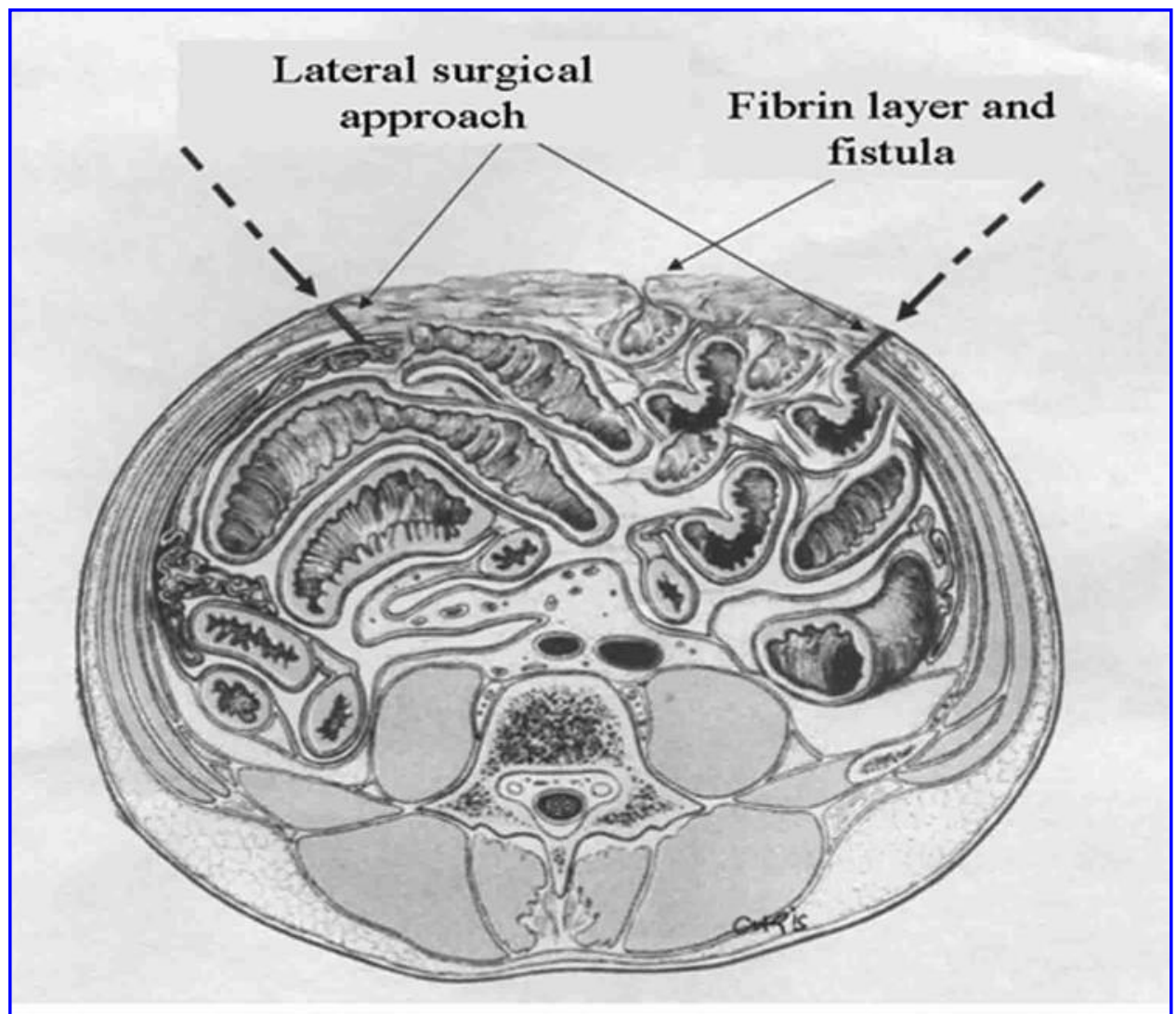

b

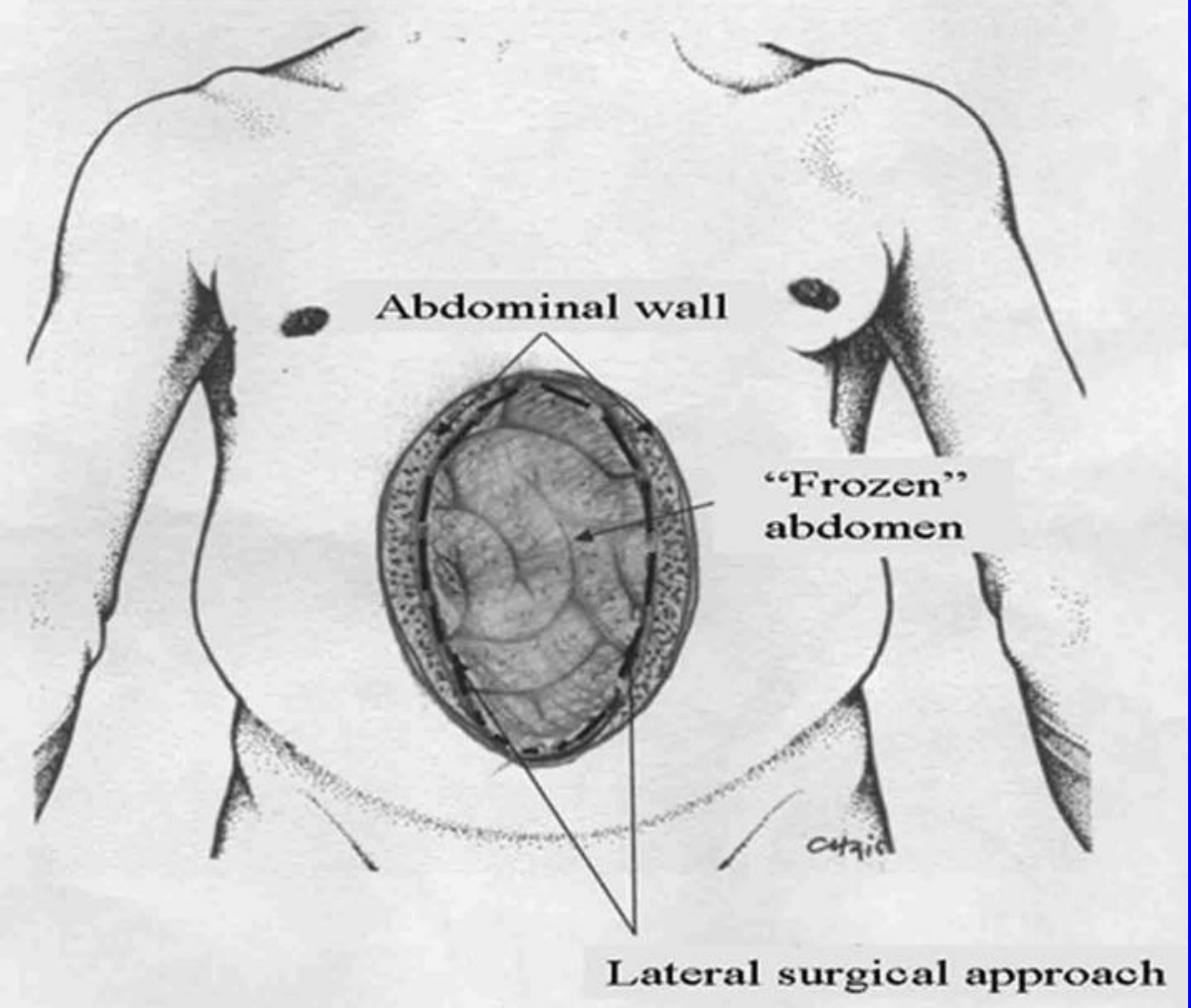

FIG. 4. (a) Lateral surgical approach is considered a safe pathway because of its avoidance of exposed viscera of "open abdomen," which is covered by fibrinous layer, and (b) safe dissection via circumference of abdominal wound and between laterally displaced abdominal wall and intra-abdominal contents. 
pist, etc.) are necessary. Decision-making is aimed at preventing exposure of adjacent abdominal viscera and organs to intestinal effluent, to control fistula output, to seal and cover the enteroatmospheric fistula, and eventually to resect the fistula tract and the responsible intestinal loop.

Conservative treatment includes bowel rest and total parenteral nutrition, appropriate administration of antibiotics to eliminate infections, ascertainment of distal bowel function, and management of the intestinal stoma and open abdomen by a senior surgeon. Thus, prevention of additional injury to the exposed abdominal viscera by limiting access to the patient's wound to one or two experienced surgeons, as was the practice in our patients, is of paramount importance.

An attempt to seal the fistula usually is unsuccessful. However, Girard et al. [3] reported an enteroatmospheric fistula that was treated successfully using a piece of acellular dermal matrix, which was secured to the stoma with the aid of fibrin glue.

Controlling fistula effluent and securing drainage far away from the adjacent viscera is important to prevent continuous inflammation and subsequent sepsis. Every effort to intubate the intestinal fistula is a grave error, resulting in the development of a larger hole. Creation of a controlled stoma with a temporary coverage plastic silo, sutured to the edges of the involved intestinal stoma, has been reported [4]. Currently, (VAC) is used widely to divert enteric flow to the device and thus avoid bowel content spillage into the peri-fistula environment, preventing continued sepsis. Application of VAC by placement of negative-pressure dressings in the surrounding abdominal wound has the additional advantage of increasing the rate of tissue granulation and augmenting wound contracture [5]. An adverse outcome is unusual, because the incidence of enteroatmospheric fistulae during management of open abdomen with VAC is about $5 \%[6,7]$. In one of our patients (case 2), the selective use of VAC promoted healing of the skin graft, contracted the wound, and protected it from enteric spillage, giving us the opportunity to seal the fistula with another grafting attempt. In another patient (case 3), in whom the fistula tract had an indirect course, application of the VAC device resulted in sealing of the fistula.

Critical appraisal of the patient's general status and local tissue conditions, as well as planning of the surgical approach to the affected intestinal loops [8], will guide the next decision. Interestingly, in our study, a lateral surgical approach was performed in two patients (cases 1 and 2). This approach consists of entering the abdominal cavity via the circumference of the open abdomen, avoiding the exposed abdominal viscera, which are covered by a fibrinous layer (Fig. 4), and safely approaching and excising the involved intestinal loop and performing an end-to-end bowel anastomosis.

Once the enteroatmospheric fistula has been resected with the involved intestinal loop or has been sealed, abdominal closure should be considered. Temporary closure with an absorbable mesh (cases 1 and 2) or with skin only (case 3) was used successively in our patients. The absorbable mesh has the advantages of covering the open abdomen and simultaneously facilitating the development of granulation tissue, which gives us the opportunity to cover the abdominal wound with autologous split-thickness skin grafts, as was the case in two of our patients (cases 1 and 2).

The psychological support of the patient and the reinforcement of intrafamilial relations must be emphasized as well. A strong physician-patient relationship anticipates and settles any emotional distress; leads to a thorough understanding of every concern the patient has for his or her body; and encourages the patient, promoting cooperation in this difficult task.

In conclusion, the surgical treatment of enteroatmospheric fistulae requires a stepwise multidisciplinary approach. An early well-planned intervention is mandatory. In our experience, a lateral surgical approach via the circumference of the open abdomen is the method of choice, facilitating resection of the involved bowel loop. Temporary abdominal coverage, preferably with an absorbable mesh, seems to be effective and permits skin grafting attempts. Finally, selective use of the VAC method is one more tool in our armamentarium.

\section{Author Disclosure Statement}

All authors state that no competing financial interests exist.

\section{References}

1. Schecter W, Ivatury R, Rotondo M, Hirshberg A. Open abdomen after trauma and abdominal sepsis: A strategy for management. J Am Coll Surg 2006;203:390-396.

2. Bosscha K, Hulstaert PF, Visser MR, et al. Open management of the abdomen and planned reoperations in severe bacterial peritonitis. Eur J Surg 2000;166:44-49.

3. Girard S, Sideman M, Spain DA. A novel approach to the problem of intestinal fistulization arising in patients managed with open peritoneal cavities. Am J Surg 2002;184:166-167.

4. Subramanian MJ, Liscum KR, Hirshberg A. The floating stoma: A new technique for controlling exposed fistulae in abdominal trauma. J Trauma 2002;53:386-388.

5. Goverman J, Yelon J, Platz JJ, et al. The "fistula-VAC," a technique of management of enterocutaneous fistulae arising within the open abdomen: Report of 5 cases. I Trauma 2006; 60:428-431.

6. Barker DE, Kaufman HJ, Smith LA, et al. Vacuum pack technique of temporary abdominal closure: A 7-year experience with 112 patients. I Trauma 2000;48:201-207.

7. Smith LA, Barker DE, Chase CW, et al. Vacuum pack technique of temporary abdominal closure: A four-year experience. Am Surg 1997;63:1102-1107.

8. Demetriades D. A technique of surgical closure of complex intestinal fistulae in the open abdomen. J Trauma 2003;55: 999-1001.

Address reprint requests to: Dr. Dionysios Voros Second Department of Surgery Aretaieion University Hospital 76 Vassilisis Sofia's Ave. 11528, Athens, Greece

E-mail: sakisdoc@yahoo.com 